\title{
X-Ray Spectroscopy Of Ion-Surface Interactions Using Superconducting Tunnel Junctions
}

\author{
T. Niedermayr ${ }^{a, c}$, S. Friedrich ${ }^{a, b}$, M. F. Cunningham ${ }^{a}$, M. Frank ${ }^{a}$, J. P. \\ Briand $^{\mathrm{c}}$ and S. E. Labov ${ }^{\mathrm{a}}$ \\ ${ }^{a}$ Lawrence Livermore National Laboratory, L-418, Livermore, CA 94551 \\ ${ }^{b}$ Lawrence Berkeley National Laboratory, MS 6-2100, Berkeley, CA 94720 \\ ${ }^{c}$ Pierre and Marie Curie University, 75005 Paris, France
}

\begin{abstract}
We have conducted low energy X-ray spectroscopy of ion/surface interactions with superconducting tunnel junction (STJ) detectors. The STJ detectors (Nb-Al-AlOx-Al-Nb) developed at the Lawrence Livermore National Laboratory have a high energy resolution and a high count-rate capability. The detectors are operated in an adiabatic demagnetization refrigerator with $60 \mathrm{mK}$ base temperature at the end of a $40 \mathrm{~cm}$ long cold finger, which can be inserted into a UHV sample chamber connected to an ion source. We have studied the interaction of $\mathrm{Ar}^{9+}, \mathrm{O}^{7+}, \mathrm{N}^{6+}$ and $\mathrm{C}^{5+}$ with various targets $\left(\mathrm{Au}, \mathrm{SiH}\right.$ and $\left.\mathrm{SiO}_{2}\right)$ at a kinetic energy of $10 \mathrm{keV} / \mathrm{q}$. The resolution of $12-15 \mathrm{eV}$ below $1 \mathrm{keV}$ allows the different satellite lines emitted by these ions to be separated.
\end{abstract}

\section{INTRODUCTION}

The interaction of ions with surfaces has been intensively researched and forms the basis of several powerful tools in modern industrial processes, especially in the area of microelectronics. The wide availability of compact multiply-charged ion (MCI) sources has made it possible to study this interaction in a new regime where the potential energy of the ion (the sum of the binding energy of the removed electrons) plays a major role in the interaction with the surface. The potential energy is deposited into the electronic system of the target material within the first few monolayers in less than $10 \mathrm{fs}$ [1], as opposed to the kinetic energy which is deposited along the tens of nanometers of the ion track. Surface modification on a nanometer scale induced by the impact of a single ion is the result of the high energy density deposited [2]. The patterning of insulator surfaces relevant for nano-technology is among the most attractive future applications of slow MCI-surface interactions.

Most of the studies to date have focused on collecting the charged and neutral particles emitted into the vacuum (electrons, ions, atoms) after ion impact on a surface. However, the large electric fields at the surface complicate the study of processes in the first few monolayers with these techniques. X-rays on the other hand are insensitive to these large electric fields and have the potential to further the understanding of the first steps of the de-excitation of the MCI [3]. A detailed knowledge of the interaction is necessary to adequately control surface modifications.

When an MCI approaches a surface, electrons are captured resonantly into states with high principal quantum number $n$. These electrons decay into lower- $n$ states by Auger 
transitions (emission of electrons into the vacuum) and/or emission of X-rays. The time spent above the surface determines how far the ion relaxes towards its neutral state before touching the surface [8]. When it finally reaches the surface, the ion will first be partially re-ionized by peeling off its loosely bound electrons, since its nuclear charge is screened by target electrons. Below the surface, inner shells are rapidly filled, followed by Auger and/or radiative transitions into the core hole. At the ion velocities used in our experiments (10 keV/q), de-excitation mainly occurs below the surface and electron capture takes place into the inner shells of the projectile.

STJ detectors offer higher energy resolution than semiconductor devices in the soft Xray range below $1 \mathrm{keV}$. This can help to resolve the different satellite lines which correspond to different electron populations in the inner shells at the time of radiative filling of the core hole. For example, in the case of the $2 p-1 \mathrm{~s}$ transition of oxygen, the satellite lines are separated by $10 \mathrm{eV}$, which cannot be resolved by a $\mathrm{Si}(\mathrm{Li})$ detector. Grating spectrometers do have a higher energy resolution, but their efficiency is lower and the need to use slits make it less advantageous in the case of an extended source of photons, as for example large ion beams interacting with surfaces.

We have used a STJ detector for low energy X-ray spectroscopy of MCI-surface interactions. We describe here the detector, the experimental setup and discuss initial results.

\section{EXPERIMENTAL SETUP}

The STJ detector used in the MCI-surface experiments was developed at the Lawrence Livermore National Laboratory for astrophysics and laboratory applications. It is described in more detail in [5]. The detectors consist of a $165 \mathrm{~nm} \mathrm{Nb}$ absorber, an Al$\mathrm{Al}_{2} \mathrm{O}_{3}$ - $\mathrm{Al}$ tunnel junction with $50 \mathrm{~nm} \mathrm{Al}$ trapping layers and a $265 \mathrm{~nm} \mathrm{Nb}$ base electrode. A room temperature FET-based preamplifier measures the excess tunneling current produced by an X-ray in the detector. We have measured an energy resolution as low as $1.7 \mathrm{eV}$ at $70 \mathrm{eV}$ and $8.9 \mathrm{eV}$ at $1 \mathrm{keV}$ using $70 \mu \mathrm{m} \times 70 \mu \mathrm{m}$ STJ detector [6]. However, here we have used a $141 \mu \mathrm{m} \times 141 \mu \mathrm{m}$ device with a resolution of $12-14 \mathrm{eV}$ at $525 \mathrm{eV}$, trading off energy resolution for increased detector area.

We operate the STJ detector in a two-stage adiabatic demagnetization refrigerator (ADR) [7]. The detector rests at the end of a $40 \mathrm{~cm}$ long cold finger, which can be inserted into a UHV chamber. The distance between the STJ detector and the target is $25 \mathrm{~mm}$. Three thin windows (200 $\AA$ aluminum with $2000 \AA$ parylene on a $90 \%$ transmissive copper grid) shield the STJ detector from IR radiation. The STJ detectors require no temperature regulation as long as they are kept below $\sim 400 \mathrm{mK}$. The ADR has a base temperature of $60 \mathrm{mK}$ with a hold time above 20 hours.

We have used a commercial Electron Cyclotron Resonance (ECR) ion source, the BIE1000 [4], to produce the MCI necessary for the experiment. This type of source offers high ion currents with moderate charge states. The source consists of a magnetically confined plasma. The production of MCI relies on microwave heating of electrons, which are accelerated to energies of tens of $\mathrm{keV}$ and ionize the surrounding gas. A $10 \mathrm{kV}$ potential extracts the ions into a UHV beamline. The ions are then charge state selected $(\mathrm{q} / \mathrm{m})$ through an analyzing magnet. After passing through a set of collimating holes (2 
$\mathrm{mm}$ diameter, $10 \mathrm{~cm}$ apart), the ions hit the sample holder under $60^{\circ}$ incidence. A manipulator allows the selection of different targets during the experiment, namely $\mathrm{H}$ passivated silicon, silicon dioxide and gold.

\section{RESULTS AND DISCUSSION}

We have studied the interaction of $\mathrm{Ar}^{9+}$ (one hole in the L-shell), $\mathrm{O}^{7+}, \mathrm{C}^{6+}$ and $\mathrm{N}^{5+}$ (one hole in the K-shell) at $10 \mathrm{keV} / \mathrm{q}$ with $\mathrm{SiH}, \mathrm{SiO}_{2}$ and $\mathrm{Au}$. The STJ detector was calibrated using $\mathrm{C} \mathrm{K}$ and $\mathrm{O} \mathrm{K}$ fluorescence from an ion gauge switched off during the subsequent experiments.
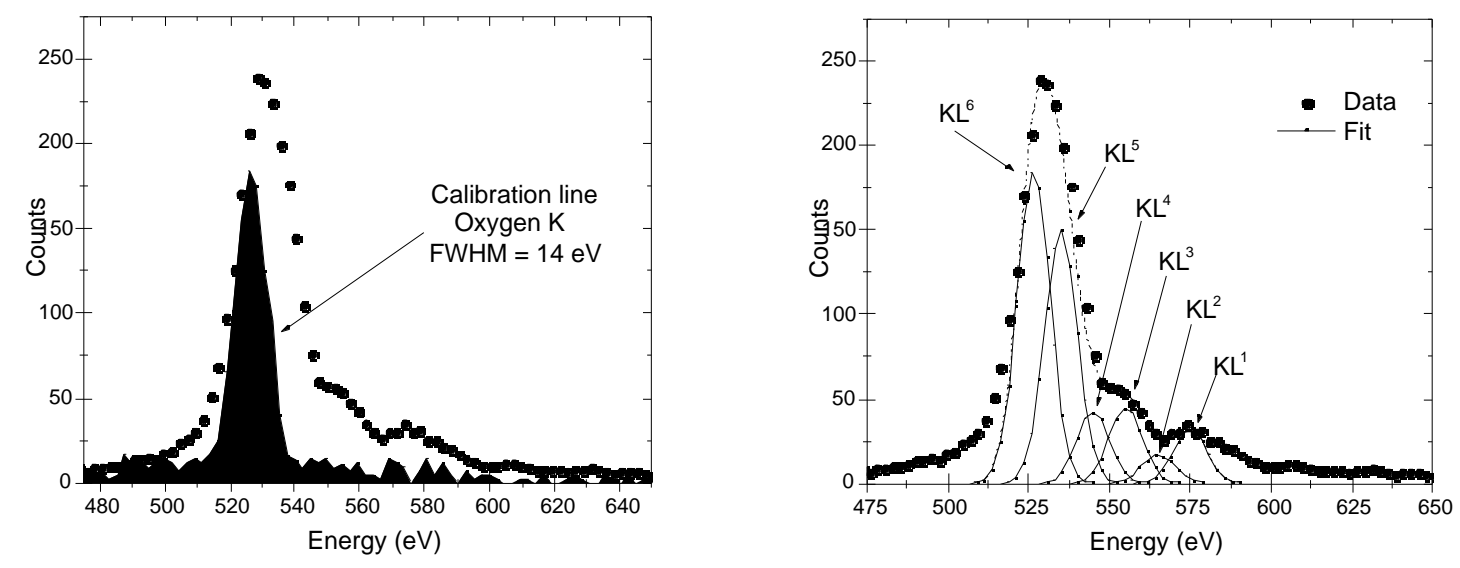

FIGURE 1: $\mathrm{X}$-ray spectra resulting from the interaction of $\mathrm{O}^{7+}$ with $\mathrm{SiH}$ at $10 \mathrm{keV} / \mathrm{q}$.

a) (left) Raw data and calibration line b) (right) Fit of the spectrum with the six satellite lines $\mathrm{KL}^{1-6}$

The resulting spectrum of the interaction of $\mathrm{O}^{7+}$ with an $\mathrm{SiH}$ surface is shown in figure 1. Spectrum a) is a superposition of the raw data with the oxygen $\mathrm{K}_{\alpha}$ calibration line $(\mathrm{FWHM}=14 \mathrm{eV})$. The spectrum of $\mathrm{O}^{7+}$ is wider than the calibration line and shows structure at higher energy. This is due to the contribution of so-called satellite lines. The $\mathrm{O} \mathrm{K}$ satellite lines arise from the dependence of the X-ray energy on the electron population of the $\mathrm{L}$ shell at the time of X-ray emission. The more electrons that are present in the L shell at the time of emission, the lower the fluorescence energy, due to screening of the nuclear charge. The 6 satellite lines of oxygen ( 6 electrons in the L shell for the neutral configuration) are separated by about $10 \mathrm{eV}$ and range from $524.9 \mathrm{eV}$ to $573.9 \mathrm{eV}$. They correspond to the $2 \mathrm{p}-1 \mathrm{~s}$ transition of $\mathrm{O}^{6+}, \mathrm{O}^{5+}, \mathrm{O}^{4+}, \mathrm{O}^{3+}, \mathrm{O}^{2+}$ and $\mathrm{O}^{1+}$. They are labeled $\mathrm{KL}^{\mathrm{x}}$, where $\mathrm{L}$ is the level from which the electron originates, $\mathrm{K}$ the level into which the electron decays and $\mathrm{x}$ the number of electrons in the $\mathrm{L}$ shell at the time of $\mathrm{X}$-ray emission. The resolution of the detector is not good enough to completely separate these lines, but it is possible to fit the data to six Gaussian lines. The energy and width of the satellite lines are known and were constrained in the fit. The sum of these six lines matches the observed spectrum (figure 1b). Each line corresponds to a different charge state of the ion. The fact that satellite lines are present is clear evidence that the ion is not fully neutralized at the time of X-ray emission. This was not predicted by current models 
of ion-surface interactions which assume that ions relaxing under the surface quickly capture electrons into the L-shell which is thus completely filled before an electron decays into the K-hole [9]. We have observed satellite lines indicative of incomplete relaxation not only for $\mathrm{O}^{7+}$ but also for $\mathrm{Ar}^{9+}, \mathrm{N}^{6+}$ and $\mathrm{C}^{5+}$. These results raise new questions about the dynamics of the neutralization inside solids.

No strong target dependency of the spectra was observed, contrary to previous experiments with decelerated $\mathrm{Ar}^{17+}$ on the same surfaces [10]. This is due to the high ion velocity used in the present experiments where the neutralization takes place below the surface instead of above. X-rays are emitted at a later time in the de-excitation process where target differences become more difficult to observe.

Future experiments will use the same ions at different kinetic energies to measure the evolution of the satellite lines with ion velocity. One can then derive the velocity dependence of the satellite line ratios and thus the evolution of the mean charge state of the ion, a first step to re-evaluate the dynamics of the de-excitation.

In summary, we have measured X-rays from ion-surface interaction experiments with a STJ detector. The good STJ detector resolution and efficiency at low X-ray energies enables us to measure the satellite lines of different ions interacting with surfaces. This demonstrates the potential of cryogenic detectors in the field of ion-surface interactions.

\section{ACKNOWLEDGMENTS}

We gratefully thank Z. Q. Xie for his valuable assistance with the ion source operation and J. D. Batteux for his expert technical work. This work was performed under the auspices of the U.S. Department of Energy by University of California Lawrence Livermore National Laboratory under contract No. W-7405-Eng-48.

\section{REFERENCES}

1. T. Schenkel et al., Phys. Rev. Lett. 83, 4273-4276 (1999)

2. G. Hayderer et al., Physica Scripta 92 156-157 (2001)

3. J.-P. Briand et al., Phys. Rev. A 54, 4136-4139 (1996)

4. Berkeley Ion Equipment, Santa Clara, CA 95054, USA.

5. M. Frank et al., Rev. Sci. Instr. 69, 25-31 (1998)

6. S. Friedrich et al., Trans. Appl. Superconductivity 9, 3330-3333 (1999)

7. S. Friedrich et al., accepted for publication in Nucl. Inst. Meth A (2001)

8. J. Burgdoerfer et al., Phys. Rev. A 44, 5674-5685 (1991)

9. J.-P. Briand et al., Phys Rev. A 55, 3947-3950 (1997)

10. J.-P. Briand et al., Phys Rev. A 55, 2526-2523 (1997) 